\title{
Avian Plasmodium in Eastern Austrian mosquitoes
}

\author{
Ellen Schoener ${ }^{1 \dagger}$, Sarah Susanne Uebleis ${ }^{1 \dagger}$, Julia Butter ${ }^{1}$, Michaela Nawratil $^{1}$, Claudia Cuk$^{1}$, Eva Flechl $^{1}$, \\ Michael Kothmayer ${ }^{1}$, Adelheid G. Obwaller ${ }^{2}$, Thomas Zechmeister ${ }^{3}$, Franz Rubel ${ }^{4}$, Karin Lebl ${ }^{4}$, Carina Zittra ${ }^{1}$ \\ and Hans-Peter Fuehrer ${ }^{1 *}$ (D)
}

\begin{abstract}
Background: Insect vectors, namely mosquitoes (Diptera: Culicidae), are compulsory for malaria parasites (Plasmodium spp.) to complete their life cycle. Despite this, little is known about vector competence of different mosquito species for the transmission of avian malaria parasites.

Methods: In this study, nested PCR was used to determine Plasmodium spp. occurrence in pools of whole individuals, as well as the diversity of mitochondrial cytochrome b gene sequences in wild-caught mosquitoes sampled across Eastern Austria in 2013-2015.

Results: A total of 45,749 mosquitoes in 2628 pools were collected, of which 169 pools (6.43\%) comprising 9 mosquito species were positive for avian Plasmodium, with the majority of positives in mosquitoes of Culex pipiens s.l./Culex torrentium. Six different avian Plasmodium lineages were found, the most common were Plasmodium vaughani SYAT05, Plasmodium sp. Linn1 and Plasmodium relictum SGS1. In 2014, mosquitoes of the Culex pipiens complex were genetically identified and Culex pipiens f. pipiens presented with the highest number of avian Plasmodium positives $(n=37 ; 16.74 \%)$. Despite this, the minimum infection rate (MIR) was highest in Culex torrentium (5.36\%) and Culex pipiens f. pipiens/f. molestus hybrids (5.26\%). During 2014 and 2015, seasonal and annual changes in Plasmodium lineage distribution were also observed. In both years P. vaughani SYAT05 dominated at the beginning of the sampling period to be replaced later in the year by P. relictum SGS1 (2014) and Plasmodium sp. Linn1 (2015).
\end{abstract}

Conclusions: This is the first large-scale study of avian Plasmodium parasites in Austrian mosquitoes. These results are of special interest, because molecular identification of the taxa of the $C$. pipiens complex and $C x$. torrentium enabled the determination of Plasmodium prevalence in the different mosquito taxa and hybrids of this complex. Since pools of whole insects were used, it is not possible to assert any vector competence in any of the examined mosquitoes, but the results are nonetheless valuable in providing an overview of avian Plasmodium species and lineages present in Austria.

\section{Background}

Haemosporidian parasites of the genus Plasmodium are responsible for avian malaria worldwide. Of the accepted 38 valid species of Plasmodium spp. [1] 488 recognised cytochrome $b$ lineages are currently described [2]. The

\footnotetext{
${ }^{*}$ Correspondence: hans-peter.fuehrer@vetmeduni.ac.at

tEllen Schoener and Sarah Susanne Uebleis contributed equally to this work

${ }^{1}$ Department of Pathobiology, Institute of Parasitology, University of Veterinary Medicine Vienna, Veterinaerplatz 1, 1210 Vienna, Austria Full list of author information is available at the end of the article
}

richest lineage diversity of Plasmodium parasites is present in South America, and in general, the diversity is much higher in tropical 'hotspot' areas (e.g., India, Australia, Southeast Asia) than in temperate regions [2]. Four very common avian Plasmodium lineages found in birds and mosquitoes in Europe are Plasmodium relictum SGS1, Plasmodium elongatum GRW6, Plasmodium vaughani SYAT05 and Plasmodium sp. Linn1 [3-6], with P. relictum SGS1 being the most common $[7,8]$. These lineages have also been found in Austria in a previous study in birds [9]. 
Avian Plasmodium parasites rely on arthropod vectors to complete their life cycle. For most species, the vectors are mosquitoes of the genera Culex, Aedes, Ochlerotatus, Culiseta and possibly Anopheles [1]. Plasmodium parasites possess a sexual and an asexual part to their life cycle; the gametogony, fertilization, formation of zygotes, and the sporogony occur in the vector, while merogony and gametocytogony happen in the vertebrate host. If the vector is fully competent, Plasmodium parasites reach and fully develop in the salivary glands, from where they are transmitted to the vertebrate host during a blood meal. The vector competence for transmitting these parasites varies between mosquito species, and each Plasmodium species may use a number of different mosquito species as vectors [10]. Not all vectors are equally susceptible to avian Plasmodium. For example, in a study comparing three vector species on Hawaii, the parasite prevalence between species varied significantly, with Culex quinquefasciatus being the most susceptible [11]. To date, a specific list of vectors for Plasmodium spp. has not yet been compiled $[1,12]$ although a list of potential vector species can be found on the MalAvi database [13]. So far, the genus Culex seems to provide the most successful vectors worldwide; in different studies it has been found that mosquitoes of this genus contained the biggest diversity of different Plasmodium strains $[10,12]$.

Forty-three indigenous species of the family Culicidae from 8 genera (Aedes, Anopheles, Culex, Coquillettidia, Culiseta, Ochlerotatus, Orthopodomyia, and Uranotaenia) have been specified in Austria [14, 15]. The establishment of the potential invasive species Aedes albopictus (Asian tiger mosquito) in Austria is still in dispute [14]. In addition, 4 more non-native species, Ochlerotatus japonicus japonicus, Anopheles hyrcanus, Orthopodomyia pulcripalpis, and Culiseta longiareolata have been reported $[14,15]$. The taxon Culex pipiens belongs to a complex and is seen in Central and Western Europe in two forms, molestus and pipiens, which frequently hybridize and cannot be distinguished morphologically [16]. Another species, Culex torrentium, while not part of this species complex, is also very difficult to distinguish morphologically [16]. All these species should therefore only be listed as individual taxa apart from the Culex pipiens species complex if they have been identified genetically.

Not all of these mosquito species are competent vectors for avian Plasmodium lineages. So far, avian Plasmodium parasites have been found in the Central European species Aedes vexans, Cx. pipiens complex, Cx. modestus, $C x$. hortensis, Culiseta annulata, Ochlerotatus caspius and the alien species Ae. albopictus [3, 17-21]. While Ae. albopictus has dramatically expanded its distribution range, this mosquito is clearly anthropophilic and has been shown to have a lower prevalence of avian malaria parasites than native Culex species [19]. Culex pipiens $\mathrm{f}$. molestus has been proven in experimental infections to be a competent vector for the $P$. relictum lineages pSGS1 and pGRW11 and GRW4 [22, 23]. While examinations of Culex pipiens f. pipiens indicate a vector role [24], they are not distinguishable from $C x$. pipiens $\mathrm{f}$. molestus. Knowledge about vector competence for most other mosquito species is currently lacking.

This is the first time that Austrian mosquitoes have been examined for avian Plasmodium on a large scale. Thousands of female mosquitoes were screened, collected over three years in three Eastern Austrian provinces, namely Vienna, Lower Austria and Burgenland for avian Plasmodium, to gain an overview which parasites are present in the area, as well as diversity and prevalence in different mosquito species. For 2014, the taxa of the $C x$. pipiens complex/Cx. torrentium were genetically identified within another project [25], which allowed to also determine avian Plasmodium incidence in morphologically similar Culex taxa in more detail.

\section{Methods}

Avian Plasmodium DNA for this study was obtained from mosquitoes sampled in two independent monitoring efforts, which also used two different storing conditions (dry and $-80^{\circ} \mathrm{C}$ ), conducted from 2013 to 2015 .

\section{Mosquito sampling effort 1}

In 2013 and 2014, adult female mosquitoes were collected by utilizing new standard miniature light traps (John W Hock Company, Gainesville, FL, USA). These traps were baited with bottled carbon dioxide (Air Liquide, Schwechat, Austria) on a daily basis for $24 \mathrm{~h}$ from March to October at three locations in Vienna. Mosquitoes were killed using the insecticide dichlorvos upon entering the trap. Once a week the traps were emptied and mosquitoes were dried and stored at room temperature until further processing.

\section{Mosquito sampling effort 2}

Mosquitoes were monitored across three provinces of Eastern Austria (Burgenland, Lower Austria, Vienna) at 35 permanent and 23 non-permanent trapping sites (Fig. 1). At permanent sampling sites, mosquitoes were collected for a 24-h period on a regular basis every 2nd week from April to October 2014-2015, using Biogents Sentinel Traps (Regensburg, Germany) equipped with bottled carbon dioxide (Air Liquide, Schwechat, Austria) as attractant. Non-permanent sampling sites were sampled at least once and up to six times during the summer months using Biogents Sentinel Traps (Regensburg, Germany) or aspirators. All mosquitoes were stored at $-80^{\circ} \mathrm{C}$ until further procedure. 


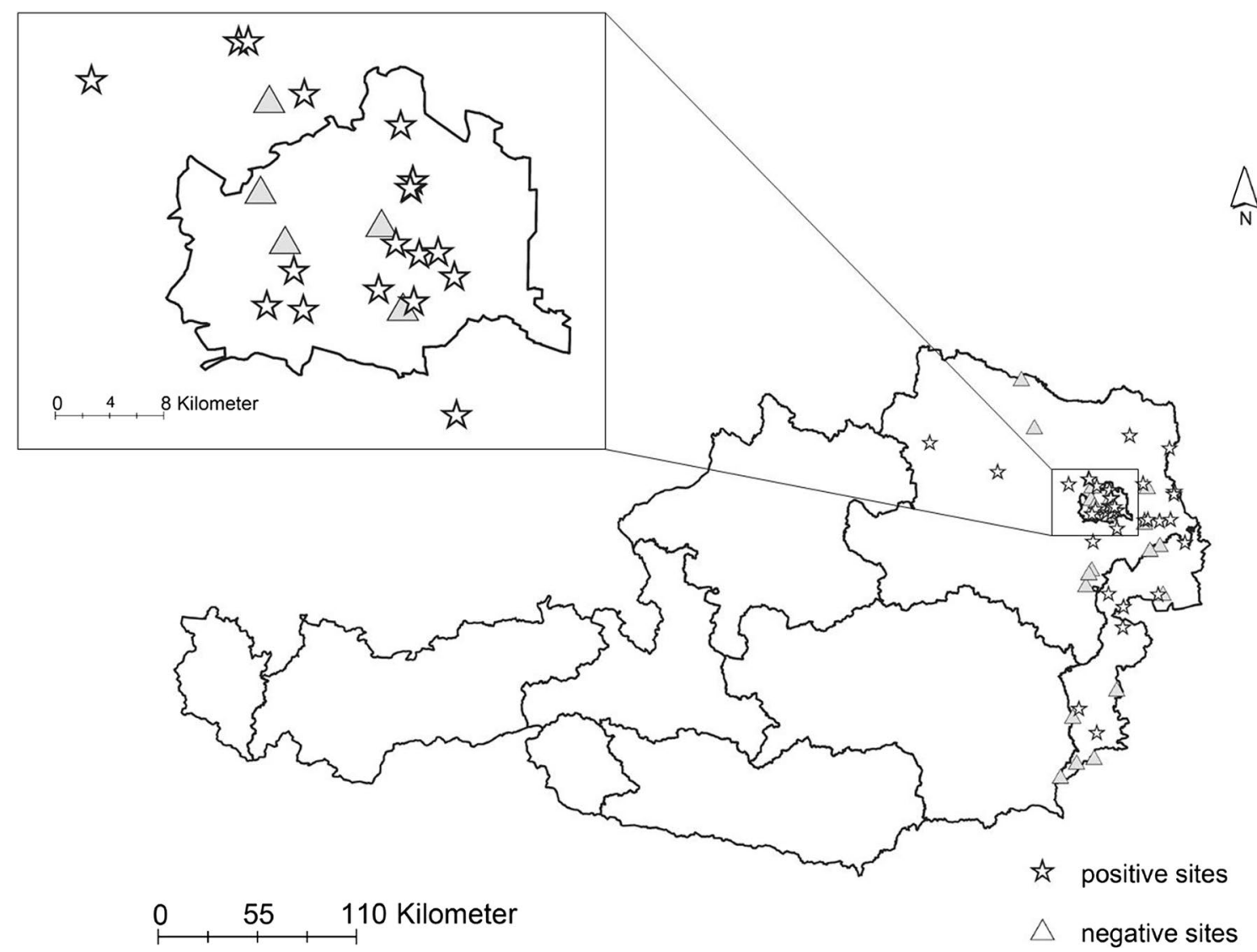

Fig. 1 Sampling sites for mosquitoes in Eastern Austria during the years 2013-2015. The close-up provides an overview of the city of Vienna where sampling sites were densest. Sites positive for avian Plasmodium are marked by stars, negative sites are marked by triangles

Morphological identification of mosquito species was performed using the identification key of Becker et al. [16]. Mosquito individuals were pooled by species, collection site and date, with a maximum number of 50 individuals (average pool size 17.9, $\min =1, \max =50$ ). To identify $C x$. pipiens taxa genetically, three legs of each individual were taken and processed individually. To each sample, $400 \mu \mathrm{l}$ of DNA/RNA lysis buffer (Zymo Research Corp., USA) and two ceramic beads (Precellys Ceramic Beads, Peqlab Biotechnologie $\mathrm{GmbH}$ ) were added and homogenized in a TissueLyser II (Qiagen, Germany). Approximately $350 \mu \mathrm{l}$ of the homogenized material was loaded onto a QIAshredder (Qiagen, Germany). To filter the samples, the filled QIAshredders were centrifuged for $2 \mathrm{~min}$ at 13,000 rpm (solid components of the samples remained on the column). DNA was extracted using a ZR-Duet ${ }^{\mathrm{TM}}$ DNA/RNA Miniprep Kit (Zymo Research Corp, USA) according to the manufacturer's instructions.

For amplifying Plasmodium spp. DNA, each DNA sample was then subjected to nested PCR, described by
[26] without modification, with the used primers targeting a $480 \mathrm{bp}$ fragment of the mitochondrial cytochrome $\mathrm{b}$ gene. PCR products were separated by electrophoresis in $2 \%$ agarose gels stained with Midori Green Advance DNA stain (Nippon Genetics Europe, Germany). Finally, purified PCR products were commercially sequenced at LGC Genomics GmbH, Germany. Obtained sequences were viewed and aligned using the programme Genious version 10.0.6 [27]. Then the sequences were compared for similarity to sequences available on the MalAvi [13] and the GenBank ${ }^{\circledR}[28]$ databases.

\section{Minimum infection rate}

To evaluate the infection rate of the collected mosquitoes, the minimum infection rate (MIR) of each mosquito species was calculated. If a mosquito pool was positive for Plasmodium on PCR, it was assumed that the pool contained at least one positive individual. Therefore, MIR (percentage) was calculated as follows:

\footnotetext{
$\operatorname{MIR}(\%)=\mathrm{n}_{(\text {PCR positive pools })} / \mathrm{n}_{(\text {total analysed mosquitoes })} \times 100$ $[5,29]$.
} 


\section{Results}

A total of 45,749 mosquitoes of 23 different species were collected in Vienna and Eastern Austria (Fig. 1) in 20132015. From these, 2628 pools were created using up to 50 whole mosquito individuals separated by species, time and site of sampling. A total of 169 (6.43\%) mosquito pools from 9 identified mosquito species and several pools of unidentified individuals of the genera Aedes/ Ochlerotatus, Culex and Anopheles were positive for avian Plasmodium. Pools positive for Plasmodium were of the following mosquito species: Ae. vexans, Coquillettidia richardii, $C x$. modestus, $C x$. pipiens complex/Cx. torrentium (unspecified to forma level), Cx. pipiens $\mathrm{f}$. pipiens, $C x$. pipiens $f$. molestus, $C x$. pipiens f. pipiens/f. molestus hybrid, $C x$. torrentium, $C x$. martinii, Ochlerotatus geniculatus, Ae. cinereus/geminus and An. plumbeus (Table 1). Avian Plasmodium was not found in the following 14 species: An. claviger, An. hyrcanus, An. maculipennis complex, Cs. annulata, Cx. territans, Oc. cantans, Oc. caspius, Oc. cataphylla, Oc. communis, Oc. flavescens, Oc. intrudens, Oc. japonicus, Oc. leucomelas and Uranotaenia unguiculata.

\section{Avian Plasmodium lineage diversity}

Six different genetic lineages of avian Plasmodium were found, with the most common $P$. vaughani SYAT05 ( $\mathrm{n}=43,25.75 \%$ of total infections), Plasmodium sp. Linn1 ( $\mathrm{n}=34,20.36 \%)$ and $P$. relictum SGS1 ( $\mathrm{n}=23$, $13.78 \%)$ and P. elongatum GRW6 $(\mathrm{n}=9,5.32 \%)$ (Table 1$)$. Only three pools $(0.11 \%)$ were positive for two other lineages, Plasmodium sp. DELURB4 and Plasmodium sp. DONANA03 (GenBank Accession Numbers: MF347696-MF347701). A total of 45 (26.95\%) pools contained more than one haemosporidian lineage, as could be seen on the electropherogram where different peaks superimposed on each other. In addition, one $C x$. pipiens pool in April 2015 was also positive for one lineage of Leucocytozoon sp. TUPHI05 (GenBank Accession Number: MF347702).

\section{Plasmodium prevalence}

In 2013, only $11(12.36 \%)$ of a total of 89 mosquito pools were detected positive for avian Plasmodium, using a sampling method which provided dried specimens stored at room temperature. Two positive pools for each Ae. cinereus/geminus, Cq. richiardii, Cx. pipiens s.l./torrentium and Oc. geniculatus were detected. Of $C x$. modestus, three pools were positive for avian Plasmodium (Table 2). Of these 11 positives, only three could be sequenced successfully, with one finding of Plasmodium sp. DONANA03 in $C x$. modestus and two in Cx. pipiens s.l./torrentium, which were mixes of several lineages with one presenting with Plasmodium sp. DONANA03 and the other with Plasmodium sp. SYAT38 as dominant lineage.

In 2014, two different sampling efforts were performed in parallel. In the first, 8404 individuals in 507 pools were collected. Of these, 10 (1.97\%) were positive for avian Plasmodium. Nine of the positives were found in Cx. pipiens s.l./torrentium, while the remaining positive was in a pool of unidentified Culex mosquitoes (Table 2). Nine of the 10 could be sequenced, presenting with two each of the lineages Plasmodium sp. Linn1 and P. vaughani SYAT05. The remaining found lineages was one of each $P$. relictum SGS1, P. elongatum GRW6 and Plasmodium sp. DONANA03.

The second, larger, sampling effort in 2014 collected 10,575 individual mosquitoes in 830 pools; of these, 53 (6.39\%) were positive for avian Plasmodium (Table 2). Of 2114 individual mosquitoes in 325 pools of $C x$. pipiens s.l./torrentium, genetic identification was performed and it was determined that $91.72 \%$ (1939 individuals in 221 pools) belonged to the subspecies $C x$. pipiens f. pipiens, $2.03 \%$ ( $n=43,26$ pools) belonged to $C x$. pipiens $\mathrm{f}$. molestus, $3.6 \%$ ( $\mathrm{n}=76,45$ pools) were hybrids of the former, and $2.65 \%$ ( $\mathrm{n}=56,33$ pools) were of the species $C x$. torrentium. Of the 325 pools in this group, $13.85 \%(n=45)$ were positive for Plasmodium (Table 3 ). The majority of Plasmodium positives were found in pools of $C x$. pipiens $\mathrm{f}$. pipiens, with $16.74 \%(\mathrm{n}=37)$ positive. Only $3.85 \%(\mathrm{n}=1)$ of Cx. pipiens f. molestus, $8.89 \%(\mathrm{n}=4)$ of the Cx. pipiens f. pipiens/f. molestus hybrids and $9.09 \%(\mathrm{n}=3)$ of $C x$. torrentium were positive (Table 2 ). Only two other identified species of mosquito were found positive for avian Plasmodium during this sampling effort in 2014, Ae. vexans and Cq. richardii. One positive pool of an unidentified lineage mix (0.66\%) was found in Ae.vexans and one pool (1.47\%) was positive for P. vaughani SYAT05 in Cq. richardii.

In 2015, 19,400 mosquito individuals, divided into 850 pools, were collected. A total of 95 pools (11.18\%) of 5 identified species and one pool of unidentified mosquitoes of the genus Anopheles and 9 pools of unidentified Culex mosquitoes were positive for avian Plasmodium (Table 3). In one pool of the Cx. pipiens complex, Leucocytozoon sp. TUPHI05 was found. The majority of positives $(\mathrm{n}=80)$ were found in $C x$. pipiens s.l./torrentium with $27.49 \%$. This group also contained the highest Plasmodium diversity, with 5 different lineages found (Linn1, SGS1, GRW11, GRW6, DELURB4). Two pools (0.92\%) of $C q$. richiardii were also positive, as well as one pool each of Ae. vexans (1.61\%), An. plumbeus (2.38\%) and $C x$. martinii (3.03\%).

\section{Minimum infection rate (MIR)}

The MIR varied between different mosquito species and between the years and different sampling events (Tables 2 


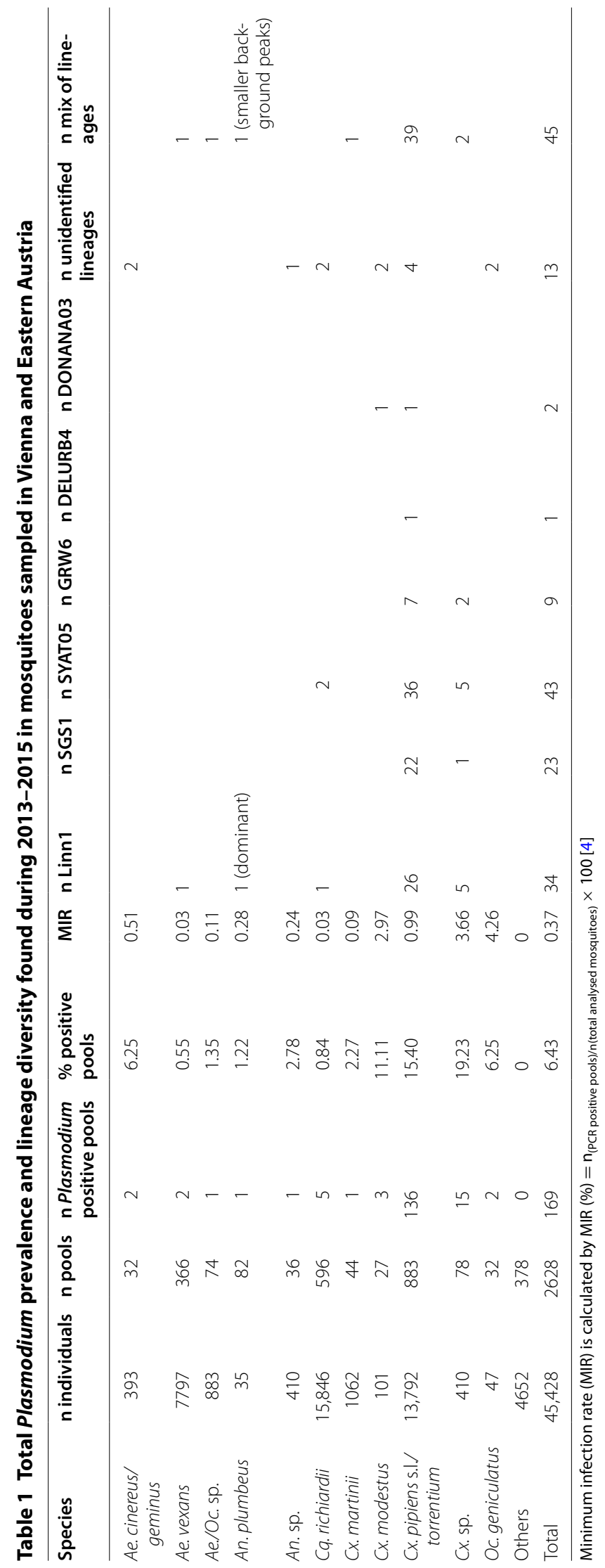




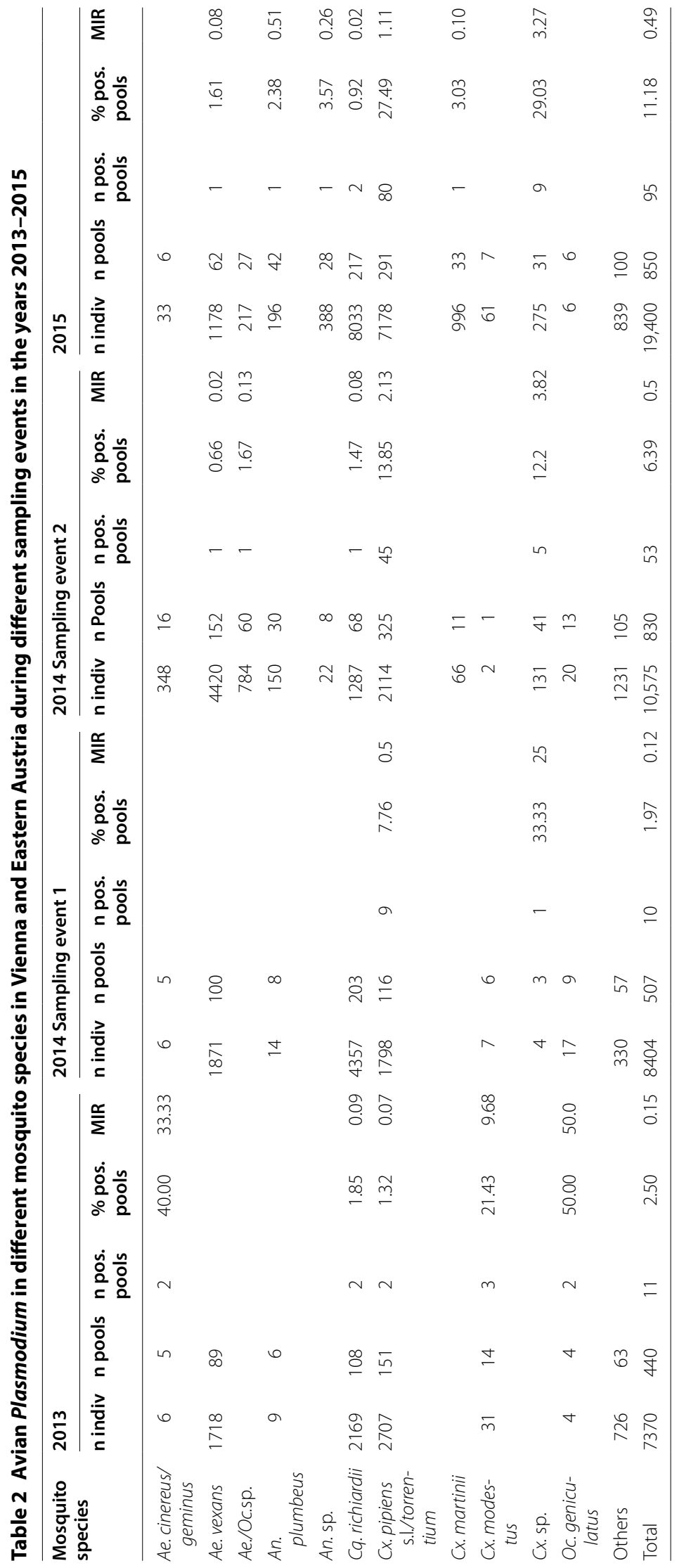




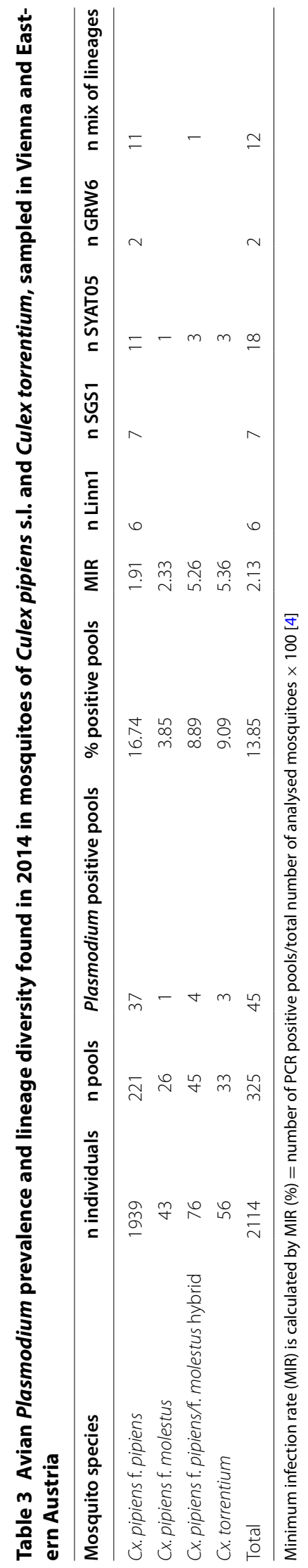


and 3, Figs. 2 and 3). The average total MIR during all years and sampling events was $0.37 \%$, with the highest averages for Oc. geniculatus (4.26\%), Cx. modestus (2.97\%) and Cx. pipiens s.l./torrentium (0.99\%) (Table 1). The highest overall MIR was found in Oc. geniculatus (50\%) and Ae. cinereus/geminus (33.33\%) in 2013 (Table 2). In 2014, where mosquitoes of the Cx. pipiens complex and $C x$. torrentium were genetically identified, it was also possible to determine the MIR in the different biotypes comprising this complex (Table 3). Here, $C x$. torrentium presented with the highest MIR (5.36\%), followed by $C x$. pipiens f. pipiens/f. molestus hybrids (5.26\%), Cx. pipiens $f$. molestus $(2.33 \%)$ and finally $C x$. pipiens f. pipiens (1.91\%).

Monthly changes and differences between 2014 and 2015 When comparing avian Plasmodium infections in different months in 2014 and 2015, only taking the second sampling event for 2014 into account (Table 2), total number and prevalence was highest in summer (August) in both years (Fig. 2). The same is true for the MIR in 2014, while the MIR in 2015 was highest in September,

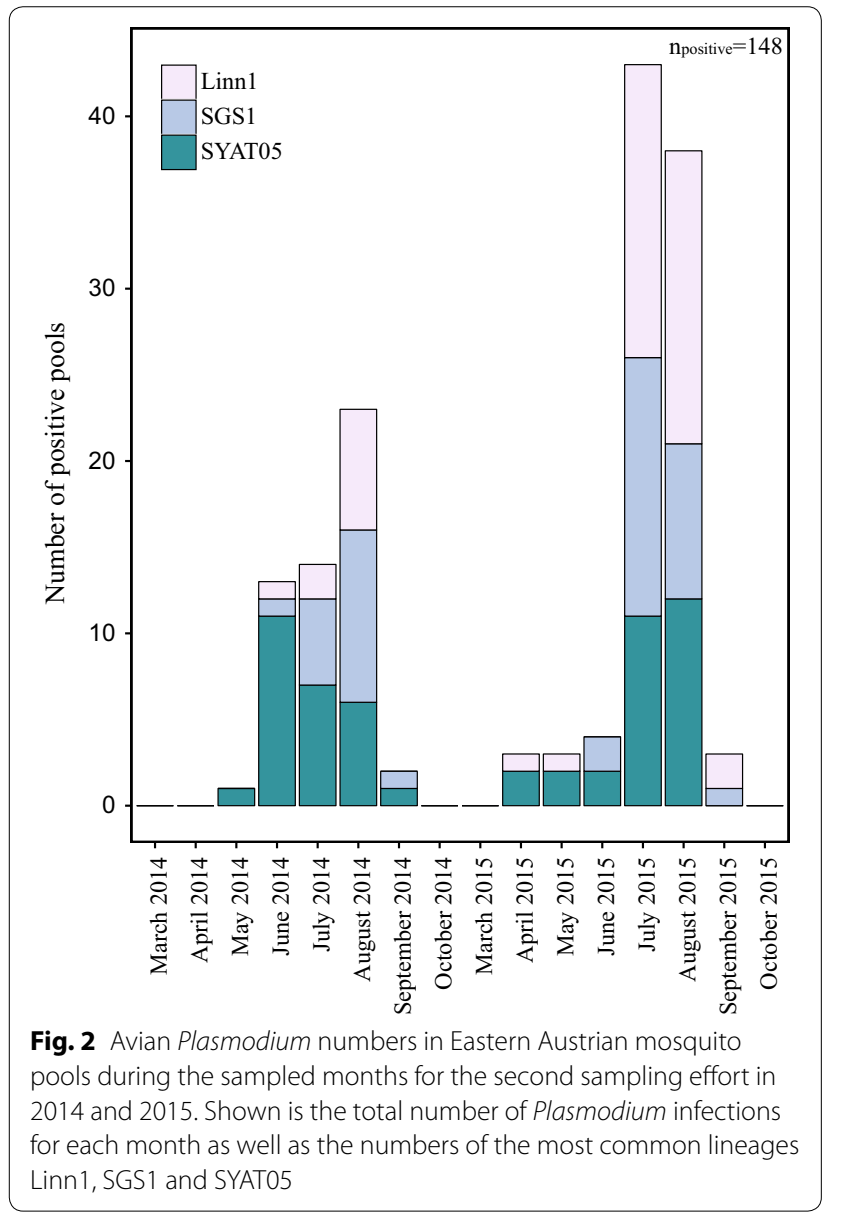

which was also the highest observed MIR of all sampled months of both years (Fig. 3). In 2014, a steep increase in the total number of infections appeared in June, while in 2015, this increase appeared 1 month later in July. Also, in 2014, the total number of infections with lineage $P$. vaughani SYAT05 was highest in June, where it dominated the lineage assembly, while later in the year, in August, $P$. relictum SGS1 became dominant (Fig. 2). In 2015, this could not be observed and the lineage Plasmodium sp. Linn1 dominated both July and August. When comparing the prevalence and MIR of different lineages over the different months (Figs. 2 and 3), P. vaughani SYAT05 was also predominant in the early sampling period (May to July) in 2014, while in August, P. relictum SGS1 dominated. Similarly, in 2015, P. vaughani SYAT05 prevalence was predominant in the beginning of the sampling period (April); but as was also seen in total number of infections in later months (July, August, September) of 2015, Plasmodium sp. Linn1 dominated.

\section{Discussion}

Plasmodium lineage diversity

This is the first large-scale study of avian Plasmodium parasites in Austrian mosquitoes. The majority of avian

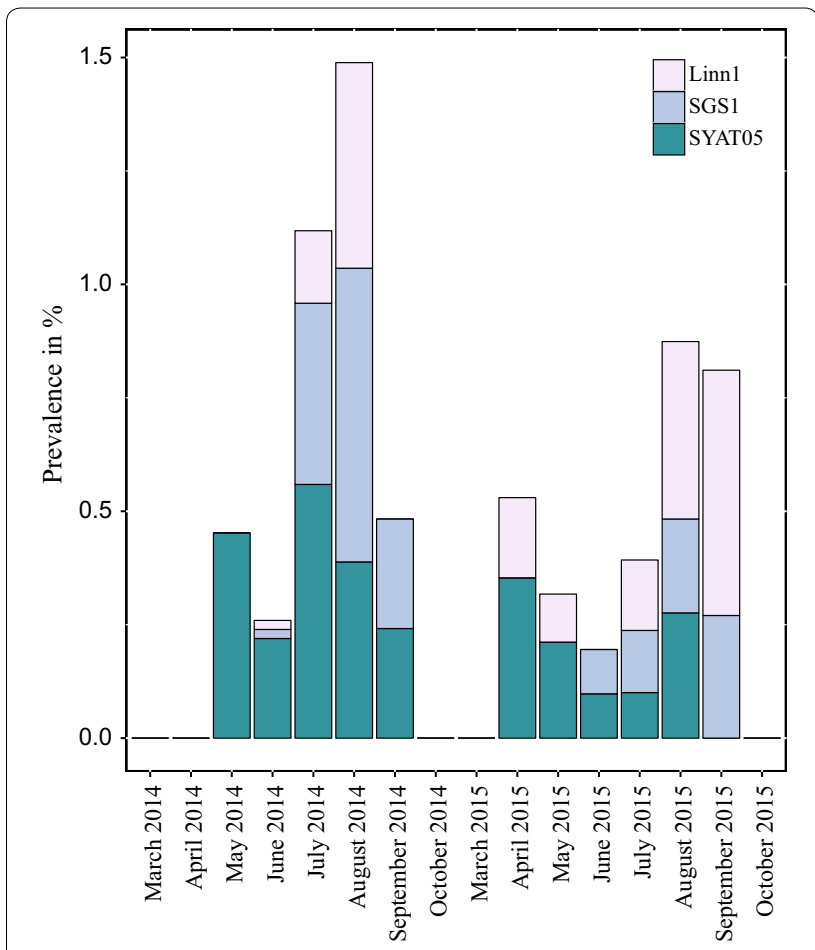

Fig. 3 Minimum infection rate (MIR) for avian Plasmodium in Eastern Austrian mosquitoes during the sampled months for the second sampling effort in 2014 and 2015. Shown is the total MIR (\%) for all Plasmodium infections as well as the MIR for the most common lineages Linn1, SGS1 and SYAT05 
Plasmodium found belonged to four very commonly detected lineages, $P$. relictum SGS1, P. elongatum GRW6, P. vaughani SYAT05 and Plasmodium sp. Linn1. These have also been reported in several previous studies on mosquito vectors in Europe. SYAT05 was found in $C x$. pipiens (L.) in the Czech Republic [30] and Switzerland [12], in Culex modestus in Spain [17] and in Cx. pipiens (L.) and Culex theileri in Portugal [5]. All three were found, among other lineages, in Cx. pipiens (L.) in France [21] and Switzerland [3]. A range of other avian malaria lineages has been found in Cx. pipiens in Italy [19], Switzerland [3], France [21], and Turkey [18].

Only a total of three of the positive pools contained the European lineages Plasmodium sp. DELURB4 and DONANA03. Since whole mosquitoes were used in pools, it was not possible to assert competent vector status on the sampled mosquito species. More than a quarter of all positive mosquito pools examined in this study carried a mix of either two or more of the most common Plasmodium lineages. Detecting mixes in this study is a by-product of examining pools of mosquitoes instead of looking at individuals, although the presence of several lineages, visible as double peaks on the chromatogram, has been found in other studies examining single mosquitoes [21]. Since mixed infections are also very common in birds [31, 32], mixed infections in individual mosquitoes should also be expected.

It can be assumed that during our study, the true diversity of avian Plasmodium in Austrian mosquitoes was greatly underestimated, a direct result of examining pooled samples where the presence of more than one parasite lineage is to be expected. Although PCR has greatly improved the detection of haemosporidian infections, it is still far from perfect. When examining infected bird blood, Jarvi et al. [33] noted that PCR diagnostics underestimated the prevalence of avian malaria in experimentally infected passerines. In addition, the acquired sequence may be of a light infection that amplifies better with the PCR protocol used, but is not identical to the parasite seen under the microscope [34]. In addition, PCR sometimes fails to amplify DNA of a clearly visible and even predominant parasite in blood samples [35]. Conventional PCR assays also underestimate mixed infections, which are common in the wild, because they might preferably amplify the DNA of one parasite over another [36].

\section{Avian Plasmodium prevalence, minimum infection rate and seasonal changes}

The overall prevalence for avian malaria found in the examined mosquito pools was $6.43 \%$. When looking at prevalence in the mosquito species, these findings are comparable with others worldwide. Two studies in
Switzerland looking at $C x$. pipiens for example found an overall prevalence of $6.6 \%(n=394)$ in $2006 / 2007$ [12] and 13.1-20.3\%, depending on the season in 2010/2011 [3]. In France, looking at the same mosquito species, prevalences ranging from $0 \%$ (February) to $15.8 \%$ (October) were observed [21]. A study in the USA, looking at different mosquito species, found $10 \%$ of 61 pools positive [37]. In studies in Japan, prevalence ranged from 14.3 to $23.9 \%$ depending on area and examined mosquito species [38-40]. Still, the result presented here is only an estimate of prevalence. When examining pooled samples, there is a chance that true prevalence is overlooked, as demonstrated in a study on mosquitoes in Vanuatu and New Caledonia [41], where total prevalence was twice when examining single individuals compared to examining pooled samples.

The MIR for different species of mosquitoes is generally very variable, and can range for example from $3.08 \%$ [39] to $0.52 \%$ [38] and $0.03 \%$ [5] in Cx. pipiens. In other species, such as Culex bitaeniorhynchus, MIR can reach as high as 6\% [42] or as high as 13\% [37]. The MIR reported in here was therefore consistent with findings in other mosquito species worldwide [37, 42, 43]. The high MIR found in Ae. cinereus/geminus and Oc. geniculatus during this study in 2013 was most likely due to the low sample size of these species collected in 2013, which skewed the result.

In general, the highest number of avian Plasmodium positives was found in late summer (August). In the northern hemisphere, most transmission of the parasites between birds occurs during the breeding season, when vector populations are increasing with the onset of warm weather. During that time, adult birds with chronic relapsing infections are available as sources of infection, and non-immune juvenile birds are hatching and leaving the nest [44]. In a study on Cx. pipiens (L.) in Switzerland, female mosquitoes caught in summer were more likely to be infected than females caught in spring [3]. Similar results were found in a study in Spain, where parasite prevalence in vectors was highest in autumn and lowest in spring [17]. In 2014 and 2015, differences were also found in dominance of several Plasmodium lineages during the examined months. In 2014, the total number of infections with lineage $P$. vaughani SYAT05 was highest in June, where it dominated the lineage assembly, while later in the year, in August, P. relictum SGS1 became dominant. In 2015, this could not be observed and the lineage Plasmodium sp. Linn1 dominated both July and August. This is comparable to findings of Labulin et al. [3], who showed that the dominance of different Plasmodium species within the studied vector population varied during the seasons. While the total prevalence increased, infections with $P$. vaughani SYAT05 decreased from 
spring to summer to be replaced by different $P$. relictum lineages SGS1, GRW11 and PADOM02. They argued that this might be due to seasonal changes in host-feeding preferences, the development of immunity in the vector against different lineages or different lineages developing under different environmental conditions.

The variations found in lineage distribution and prevalence comparing different years may be explained with an annual variation in vector abundance and activity. The feeding activity of dipteran vectors depends on weather conditions and can be affected by temperature and wind. Another explanation for the annual changes might be the winter mortality of the vertebrate hosts. Blood parasites may cause pathology in the host $[9,45]$, and during winter, uninfected birds may survive better than infected ones [46].

In general, samples collected with method 1 in 2013 and 2014 and stored as dry specimens yielded far less positives than samples collected with method 2 and it is likely that the DNA quality was compromised by the storage method, as shown in a previous study by Werblow et al. [47].

\section{Avian Plasmodium in mosquitoes of the Culex pipiens complex s.l./Culex torrentium}

The second-most common mosquitoes caught in this study were taxa belonging to the morphologically indistinguishable $C x$. pipiens complex and $C x$. torrentium. During a previous study, mosquitoes of this species complex sampled in 2014 were identified genetically [16] and this provided an opportunity to determine avian Plasmodium diversity and prevalence in these dipterans. During sampling, the most common mosquito of this species group caught was $C x$. pipiens f. pipiens and subsequently, the largest total number of avian Plasmodium parasites as well as the largest proportion of positive pools was found in this species. However, when comparing the MIR of the different species and the hybrids in the species complex, differences are evident. Culex torrentium showed the highest MIR, followed by the Cx. pipiens $\mathrm{f}$. pipiens/f. molestus hybrids, while the MIR for Cx. pipiens was lowest. It is unclear if these differences could be explained by the much lower sample size of $C x$. pipiens $\mathrm{f}$. molestus, $C x$. torrentium and hybrids or if these mosquitoes in general bite birds more frequently and therefore have a higher chance of acquiring avian Plasmodium.

In general, mosquitoes of this species complex provided the majority of avian Plasmodium detected during this study. This finding is not surprising, since the genus Culex provides the most successful vectors for these parasites worldwide $[10,12]$. In addition, mosquitoes of the $C x$. pipiens complex and their hybrids frequently bite birds [6] and have therefore the opportunity to acquire avian Plasmodium with a blood meal.

One lineage of Leucocytozoon sp. TUPHI05 in one pool of undetermined mosquitoes of the species complex was also detected. Members of the genus Leucocytozoon are haemosporidian parasites, which are closely related to Plasmodium and also infect birds, although they are transmitted by black flies of the family Simuliidae [1]. It is not unusual to find haemosporidian parasites in vectors that usually do not transmit them, especially when whole insects are examined and vector competence cannot be asserted [6].

\section{Conclusions}

To gain further insight into avian Plasmodium prevalence, distribution and diversity in mosquitoes in Austria, further research is required. New molecular detection methods are needed to reliably and simultaneously detect different lineages in a single sample. For a better understanding of parasite transmission and vector competence, experimental infections as well as the examination of mosquito thoraxes and salivary glands for avian Plasmodium are needed. Examining individual mosquitoes would be ideal for detecting the real diversity of Plasmodium parasites $[10,21]$, but this approach is costly and many research groups use pooled samples [40, 48, 49]. In the case of blood-fed mosquitoes, individuals have to be processed individually to determine the bitten bird host $[40,50]$. It is high time to carry out such studies now, as data collected in the near future will provide the basis for the evaluation of long-term changes to the system birdvector-parasite due to human impacts (changes and/or destruction of bird habitat) and changes in bird and vector distributions due to predicted climate change.

\section{Authors' contributions}

ES and HPF wrote the initial draft of the manuscript. The study was designed and supervised by HPF. Mosquito collection (including organization) was performed by HPF, AGO, TZ, FR, KL, and CZ. Morphological mosquito species identification was performed by $\mathrm{CZ}$ and KL. Molecular mosquito specification was performed by EF and MK. Molecular pathogen assays and sequence analyses were performed by SSU, JB, MN, CC, ES, and HPF. All authors read and approved the final manuscript.

\section{Author details \\ ${ }^{1}$ Department of Pathobiology, Institute of Parasitology, University of Veteri- nary Medicine Vienna, Veterinaerplatz 1, 1210 Vienna, Austria. ${ }^{2}$ Division of Sci- ence, Research and Development, Federal Ministry of Defence and Sports, Vienna, Austria. ${ }^{3}$ Biological Station Lake Neusiedl, Burgenland, Austria. ${ }^{4}$ Department for Farm Animals and Veterinary Public Health, Institute for Vet- erinary Public Health, University of Veterinary Medicine Vienna, Veterinaerplatz 1, 1210 Vienna, Austria.}

\section{Acknowledgements}

We thank all citizen scientists who helped at mosquito sampling within this study.

\section{Competing interests}

The authors declare that they have no competing interests. 


\section{Availability of data and materials}

The data supporting the conclusions of this article are provided within the article. The sequences are submitted in the GenBank database under Accession Numbers MF347696-MF347702

\section{Consent for publication}

Not applicable.

\section{Ethics approval}

Not applicable.

\section{Funding}

This research was funded by the ERA-Net BiodivERsA, with the National Funders FWF 1-1437, ANR-13-EBID-0007-01 and DFG BiodivERsA KL 2087/6-1 as part of the 2012-13 BiodivERsA call for research proposals.

\section{Publisher's Note}

Springer Nature remains neutral with regard to jurisdictional claims in published maps and institutional affiliations.

Received: 29 June 2017 Accepted: 19 September 2017 Published online: 29 September 2017

\section{References}

1. Valkiunas $\mathrm{G}$, editor. Avian malaria parasites and other Haemosporidia. Boca Raton: CRC; 2005.

2. Clark NJ, Clegg SM, Lima MR. A review of global diversity in avian haemosporidians (Plasmodium and Haemoproteus: Haemosporida): new insights from molecular data. Int J Parasitol. 2014;44:329-38.

3. Lalubin F, Deledevant A, Glaizot O, Christe P. Temporal changes in mosquito abundance (Culex pipiens), avian malaria prevalence and lineage composition. Parasit Vectors. 2013;6:307.

4. Marzal A, Bensch S, Reviriego M, Balbontin J, de Lope F. Effects of malaria double infection in birds: one plus one is not two. J Evol Biol. 2008;21:979-87.

5. Ventim R, Ramos JA, Osorio H, Lopes RJ, Perez-Tris J, Mendes L. Avian malaria infections in western European mosquitoes. Parasitol Res. 2012;111:637-45

6. la Puente Martínez-de, Ferraguti M, Ruiz S, Roiz D, Soriguer RC, Figuerola J. Culex pipiens forms and urbanization: effects on blood feeding sources and transmission of avian Plasmodium. Malar J. 2016;15:589.

7. Hellgren O, Perez-Tris J, Bensch S. A jack-of-all-trades and still a master of some: prevalence and host range in avian malaria and related blood parasites. Ecology. 2009:90:2840-9.

8. Hellgren O, Atkinson CT, Bensch S, Albayrak T, Dimitrov D, Ewen JG, et al Global phylogeography of the avian malaria pathogen Plasmodium relictum based on MSP1 allelic diversity. Ecography. 2015;38:842-50.

9. Dinhopl N, Nedorost N, Mostegl MM, Weissenbacher-Lang C, Weissenbock $\mathrm{H}$. In situ hybridization and sequence analysis reveal an association of Plasmodium spp. with mortalities in wild passerine birds in Austria. Parasitol Res. 2015;114:1455-62.

10. Kimura M, Darbro JM, Harrington LC. Avian malaria parasites share congeneric mosquito vectors. J Parasitol. 2010;96:144-51.

11. LaPointe DA, Goff ML, Atkinson CT. Comparative susceptibility of introduced forest dwelling mosquitoes in Hawai'i to avian malaria, Plasmodium relictum. J Parasitol. 2005;91:843-9.

12. Glaizot O, Fumagalli L, Iritano K, Lalubin F, Van Rooyen J, Christe P. High prevalence and lineage diversity of avian malaria in wild populations of great tits (Parus major) and mosquitoes (Culex pipiens). PLOS ONE. 2012; $7:$ e34964.

13. Bensch S, Hellgren O, Perez-Tris J. MalAvi. A public database of malaria parasites and related haemosporidians in avian hosts based on mitochondrial cytochrome b lineages. Mol Ecol Res. 2009;9:1353-8.

14. Zittra C, Joachim A, Fuehrer H-P. Mosquitoes and Dirofilaria in Austria-a review of the current situation of neobiotic Culicidae and Dirofilariae. Tierärztliche Umschau. 2015:70:126-31.
15. Zittra C, Obwaller AG, Wimmer V, Berer D, Eigner B, Fuehrer HP. First record of Orthopodomya pulcripalpis (Rondani, 1872) (Diptera: Culicidae) in Austria. Parasitol Res. 2017;116:1781-3.

16. Becker N, Petrić D, Boase C, Lane J, Zgomba M, Dahl C, Kaiser A. Mosquitoes and their control. Berlin: Springer; 2003.

17. Ferraguti M, Martinez-de la Puente J, Munoz J, Roiz D, Ruiz S, Soriguer R, Figuerola J. Avian Plasmodium in Culex and Ochlerotatus mosquitoes from Southern Spain: effects of season and host-feeding source on parasite dynamics. PLoS ONE. 2013;8:e66237.

18. Inci A, Yildirim A, Njabo K, Duzlu O, Biskin Z, Ciloglu A. Detection and molecular characterization of avian Plasmodium from mosquitoes in central Turkey. Vet Parasitol. 2012;188:179-84.

19. Martinez-de la Puente J, Munoz J, Capelli G, Montarsi F, Soriguer R, Arnoldi D, et al. Avian malaria parasites in the last supper: identifying encounters between parasites and the invasive Asian mosquito tiger and native mosquito species in Italy. Malar J. 2015;14:32.

20. Santiago-Alarcon D, Palinauskas V, Schaefer HM. Diptera vectors of avian Haemosporidian parasites: untangling parasite life cycles and their taxonomy. Biol Rev. 2012;87:928-64.

21. Zele F, Vezilier J, L'Ambert G, Nicot A, Gandon S, Rivero A, et al. Dynamics of prevalence and diversity of avian malaria infections in wild Culex pipiens mosquitoes: the effects of Wolbachia, filarial nematodes and insecticide resistance. Parasit Vectors. 2014;7:437.

22. Ziegyte R, Bernotiene R, Bukauskaite D, Palinauskas V, lezhova T, Valkiunas $G$. Complete sporogony of Plasmodium relictum (lineages pSGS1 and pGRW11) in mosquito Culex pipiens pipiens form molestus, with implications to avian malaria epidemiology. J Parasitol. 2014;100:878-82

23. Valkiunas $G$, Ziegyte $R$, Palinauskas V, Bernotiene R, Bukauskaite D, Ilgunas $M$, et al. Complete sporogony of Plasmodium relictum (lineage pGRW4) in mosquitoes Culex pipiens pipiens, with implications on avian malaria epidemiology. Parasitol Res. 2015;114:3075-85.

24. Kazlauskiene R, Bernotiene R, Palinauskas V, lezhova TA, Valkiunas G. Plasmodium relictum (lineages PSGS1 and pGRW11): complete synchronous sporogony in mosquitoes Culex pipiens pipiens. Exp Parasitol. 2013;133:454-61.

25. Zittra C, Flechl E, Kothmayer M, Vitecek S, Rossiter H, Zechmeister T, et al. Ecological characterization and molecular differentiation of Culex pipiens complex taxa and Culex torrentium in eastern Austria. Parasit Vectors. 2016;9:197.

26. Hellgren $\mathrm{O}$, Waldenstrom J, Bensch S. A new PCR assay for simultaneous studies of Leucocytozoon, Plasmodium, and Haemoproteus from avian blood. J Parasitol. 2004;90:797-802.

27. Kearse M, Moir R, Wilson A, Stones-Havas S, Cheung M, Sturrock S, Buxton $S$, et al. Geneious Basic: an integrated and extendable desktop software platform for the organization and analysis of sequence data. Bioinformatics. 2012;28:1647-9.

28. Benson DA, Karsch-Mizrachi I, Lipman DJ, Ostell J, Sayers EW. GenBank. Nucleic Acids Res. 2011;39:D32-7.

29. White BJ, Andrew DR, Mans NZ, Ohajuruka OA, Garvin MC. West Nile Virus in mosquitoes of northern Ohio, 2003. Am J Trop Med Hyg. 2006;75:346-9.

30. Synek P, Munclinger P, Albrecht T, Votypka J. Avian haemosporidians in haematophagous insects in the Czech Republic. Parasitol Res. 2013:112:839-45.

31. Jarvi SI, Farias ME, Atkinson CT. Genetic characterization of Hawaiian isolates of Plasmodium relictum reveals mixed-genotype infections. Biol Direct. 2008;3:25

32. Van Rooyen J, Lalubin F, Glaizot O, Christe P. Avian haemosporidian persistence and co-infection in great tits at the individual level. Malar J. 2013;12:40

33. Jarvi SI, Schultz JJ, Atkinson CT. PCR diagnostics underestimate the prevalence of avian malaria (Plasmodium relictum) in experimentally-infected passerines. J Parasitol. 2002:88:153-8.

34. Valkiūnas $G$, Zehtindjiev $P$, Hellgren $O$, Ilieva M, lezhova TA, Bensch S. Linkage between mitochondrial cytochrome $b$ lineages and morphospecies of two avian malaria parasites, with a description of Plasmodium (Novyella) ashfordi sp. nov. Parasitol Res. 2007;100:1311-22. 
35. Zehtindjiev P, Krizanauskiene A, Bensch S, Palinauskas V, Asghar M, Dimitrov $D$, et al. A new morphologically distinct avian malaria parasite that fails detection by established polymerase chain reaction-based protocols for amplification of the cytochrome b gene. J Parasitol. 2012;98:657-65.

36. Valkiunas G, Bensch S, lezhova TA, Krizanauskiene A, Hellgren O, Bolshakov CV. Nested cytochrome B polymerase chain reaction diagnostics underestimate mixed infections of avian blood haemosporidian parasites: microscopy is still essential. J Parasitol. 2006;92:418-22.

37. Fryxell RTT, Lewis TT, Peace H, Hendricks BBM, Paulsen D. Identification OF avian malaria (Plasmodium sp.) and canine heartworm (Dirofilaria immitis) in the mosquitoes of Tennessee. J Parasitol. 2014:100:455-62.

38. Ejiri H, Sato Y, Sawai R, Sasaki E, Matsumoto R, Ueda M, et al. Prevalence of avian malaria parasite in mosquitoes collected at a zoological garden in Japan. Parasitol Res. 2009;105:629-33.

39. Kim KS, Tsuda Y. Seasonal changes in the feeding pattern of Culex pipiens pallens govern the transmission dynamics of multiple lineages of avian malaria parasites in Japanese wild bird community. Mol Ecol. 2010;19:5545-54.

40. Kim KS, Tsuda Y, Yamada A. Bloodmeal identification and detection of avian malaria parasite from mosquitoes (Diptera: Culicidae) inhabiting coastal areas of Tokyo Bay, Japan. J Med Entomol. 2009;46:1230-4.

41. Ishtiaq F, Guillaumot L, Clegg S, Phillimore A, Black R, Owens I, Mundy N, et al. Avian haematozoan parasites and their associations with mosquitoes across Southwest Pacific Islands. Mol Ecol. 2008;17:4545-55.

42. Kim KS, Tsuda Y. Avian Plasmodium lineages found in spot surveys of mosquitoes from 2007 to 2010 at Sakata wetland, Japan: do dominant lineages persist for multiple years? Mol Ecol. 2012;21:5374-85.
43. Ejiri H, Sato Y, Kim K-S, Hara T, Tsuda Y, Imura T, et al. Entomological study on transmission of avian malaria parasites in a zoological garden in Japan: bloodmeal identification and detection of avian malaria parasite DNA from blood-fed mosquitoes. J Med Entomol. 2011;48:600-7.

44. Atkinson CT, van Riper C. Pathogenicity and epizootiology of avian haematozoa: Plasmodium, Leucocytozoon, and Haemoproteus. In: Loye JE, Zuk M, editors. Bird-parasite interactions: ecology, evolution, and behavior. Oxford: Oxford University Press; 1991.

45. Ilgūnas M, Bukauskaitè D, Palinauskas V, lezhova TA, Dinhopl N, Nedorost $\mathrm{N}$, et al. Mortality and pathology in birds due to Plasmodium (Giovannolaia) homocircumflexum infection, with emphasis on the exoerythrocytic development of avian malaria parasites. Malar J. 2016;15:256.

46. Allander K, Bennett GF. Prevalence and intensity of haematozoan infection in a population of great tits Parus major from Gotland, Sweden. J Avian Biol. 1994;25:69-74.

47. Werblow A, Flechl E, Klimpel S, Zittra C, Lebl K, Kieser K. Direct PCR of indigenous and invasive mosquito species: a time-and cost-effective technique of mosquito barcoding. Med Vet Entomol. 2016;30:8-13.

48. Njabo KY, Cornel AJ, Bonneaud C, Toffelmier E, Sehgal RNM, Valkiūnas $\mathrm{G}$, et al. Nonspecific patterns of vector, host and avian malaria parasite associations in a central African rainforest. Mol Ecol. 2011;20:1049-61.

49. Njabo KY, Cornel AJ, Sehgal RNM, Loiseau C, Buermann W, Harrigan RJ, et al. Coquillettidia (Culicidae, Diptera) mosquitoes are natural vectors of avian malaria in Africa. Malar J. 2009;8:193.

50. Hamer GL, Kitron UD, Goldberg TL, Brawn JD, Loss SR, Ruiz MO, et al. Host selection by Culex pipiens mosquitoes and West Nile virus amplification. Am J Trop Med Hyg. 2009;80:268-78.

\section{Submit your next manuscript to BioMed Central and we will help you at every step:}

- We accept pre-submission inquiries

- Our selector tool helps you to find the most relevant journal

- We provide round the clock customer support

- Convenient online submission

- Thorough peer review

- Inclusion in PubMed and all major indexing services

- Maximum visibility for your research

Submit your manuscript at www.biomedcentral.com/submit
() Biomed Central 